Article

\title{
Exploring Deep Physiological Models for Nociceptive Pain Recognition
}

\author{
Patrick Thiam ${ }^{1,2, *}$, Peter Bellmann ${ }^{2}$, Hans A. Kestler ${ }^{1}\left(\mathbb{D}\right.$ and Friedhelm Schwenker ${ }^{2}$ \\ 1 Institute of Medical Systems Biology, Ulm University, Albert-Einstein-Allee 11, 89081 Ulm, Germany; \\ hans.kestler@uni-ulm.de \\ 2 Institute of Neural Information Processing, Ulm University, James-Franck-Ring, 89081 Ulm, Germany; \\ peter.bellmann@uni-ulm.de (P.B.); friedhelm.schwenker@uni-ulm.de (F.S.) \\ * Correspondence: patrick.thiam@uni-ulm.de
}

Received: 5 September 2019; Accepted: 14 October 2019; Published: 17 October 2019

check for updates

\begin{abstract}
Standard feature engineering involves manually designing measurable descriptors based on some expert knowledge in the domain of application, followed by the selection of the best performing set of designed features for the subsequent optimisation of an inference model. Several studies have shown that this whole manual process can be efficiently replaced by deep learning approaches which are characterised by the integration of feature engineering, feature selection and inference model optimisation into a single learning process. In the following work, deep learning architectures are designed for the assessment of measurable physiological channels in order to perform an accurate classification of different levels of artificially induced nociceptive pain. In contrast to previous works, which rely on carefully designed sets of hand-crafted features, the current work aims at building competitive pain intensity inference models through autonomous feature learning, based on deep neural networks. The assessment of the designed deep learning architectures is based on the BioVid Heat Pain Database (Part A) and experimental validation demonstrates that the proposed uni-modal architecture for the electrodermal activity (EDA) and the deep fusion approaches significantly outperform previous methods reported in the literature, with respective average performances of $84.57 \%$ and $84.40 \%$ for the binary classification experiment consisting of the discrimination between the baseline and the pain tolerance level ( $T_{0} \mathrm{vs}$. $T_{4}$ ) in a Leave-One-Subject-Out (LOSO) cross-validation evaluation setting. Moreover, the experimental results clearly show the relevance of the proposed approaches, which also offer more flexibility in the case of transfer learning due to the modular nature of deep neural networks.
\end{abstract}

Keywords: convolutional neural networks; signal processing; information fusion; pain intensity classification

\section{Introduction}

Conventional machine learning approaches are built upon a set of carefully engineered representations, which consist of measurable parameters extracted from raw data. Based on some expert knowledge in the domain of application, a feature extractor is designed and used to extract relevant information in the form of a feature vector from the preprocessed raw data. This high level representation of the input data is subsequently used to optimise an inference model. Although such approaches have proven to be very effective and can potentially lead to state-of-the-art results (given that the set of extracted descriptors is suitable for the task at hand), the corresponding performance and generalisation capability is limited by the reliance on expert knowledge as well as the inability of the designed model to process raw data directly and to dynamically adapt to related new tasks. 
Meanwhile, deep learning approaches [1] automatically generate suitable representations by applying a succession of simple and non-linear transformations on the raw data. A deep learning architecture consists of a hierarchical construct of several processing layers. Each processing layer is characterised by a set of parameters that are used to transform its input (which is the representation generated by the previous layer) into a new and more abstract representation. This specific hierarchical combination of several non-linear transformations enables deep learning architectures to learn very complex functions as well as abstract descriptive (or discriminative) representations directly from raw data [2]. Moreover, the hierarchical construct characterising deep learning architectures offers more flexibility when it comes to adapting such approaches to new and related tasks. Hence, deep learning approaches have been outperforming previous state-of-the-art machine learning approaches, especially in the field of image processing [3-7]. Similar performances have been achieved in the field of speech recognition [8,9] and natural language processing $[10,11]$.

A steadily growing amount of work has been exploring the application of deep learning approaches on physiological signals. Martinéz et al. [12] were able to significantly outperform standard approaches built upon hand-crafted features by using a deep learning algorithm for affect modelling based on physiological signals (two physiological signals consisting of Skin Conductance (SC) and Blood Volume Pulse (BVP) were used in this specific work). The designed approach consisted of a multi-layer Convolutional Neural Network (CNN) [13] combined with a single-layer perceptron (SLP). The parameters of the CNN were trained in an unsupervised manner using denoising auto-encoders [14]. The SLP was subsequently trained in a supervised manner using backpropagation [15] to map the outputs of the CNN to the target affective states. In [16], the authors proposed a multiple-fusion-layer based ensemble classifier of stacked auto-encoder (MESAE) for emotion recognition based on physiological data. A physiological-data-driven approach was proposed in order to identify the structure of the ensemble. The architecture was able to significantly outperform the existing state-of-the-art performance. A deep CNN was also successfully applied in [17] for arousal and valence classification based on both electrocardiogram (ECG) and Galvanic Skin Response (GSR) signals. In [18], a hybrid approach using CNN and Long Short-Term Memory (LSTM) [19] Recurrent Neural Network (RNN) was designed to automatically extract and merge relevant information from several data streams stemming from different modalities (physiological signals, environmental and location data) for emotion classification. Moreover, deep learning approaches have been applied on electromyogram (EMG) signals for gesture recognition $[20,21]$ or hand movement classification [22,23]. Most of the reported approaches consist of first transforming the processed EMG signal into a two dimensional (time-frequency) visual representation (such as a spectrogram or a scalogram) and subsequently using a deep CNN architecture to proceed with the classification. A similar procedure was used in [24] for the analysis of electroencephalogram (EEG) signals. These are just some examples of an increasingly growing field of experimentation for deep neural networks. A better overview of deep learning approaches applied to physiological signals can be found in $[25,26]$. However, there are few related works that focus specifically on the application of deep neural networks on physiological signals for pain recognition. The authors of [27] recently proposed a classification architecture based on Deep Belief Networks (DBNs) for the assessment of patients' pain level during surgery, using photoplethysmography (PPG). The proposed architecture consists of a bagged ensemble of DBNs, built upon a set of manually engineered features, extracted from the recorded and preprocessed PPG signals. It is important to note that, in this specific study, the ensemble of bagged DBNs was trained on a set of carefully designed hand-crafted features. Therefore, an expert knowledge in this specific area of application is still needed in order to generate a set of relevant descriptors, since the whole classification process is not performed in an end-to-end manner.

Nonetheless, there is a constantly growing amount of works that focus specifically on pain recognition based on physiological signals, and categorised by the nature of the pain elicitations. There is a huge 
variety of statistical methods that have been proposed, most of them based on more traditional machine learning approaches such as decision trees or Support Vector Machines (SVMs) [28]. In [29], the authors proposed a continuous pain monitoring method using an Artificial Neural Network (ANN), based on hand-crafted features (wavelength (WL) and root mean square (RMS) features) extracted from several physiological signals consisting of heart rate (HR), breath rate (BR), galvanic skin response (GSR) and facial surface electromyogram (sEMG). The proposed approach was assessed on a dataset collected by inducing both thermal and electrical pain stimuli. In [30], the authors proposed a pain detection approach based on EEG signals. Relevant features are extracted from the EEG signals using the Choi-Williams quadratic time-frequency distribution and subsequently used to train a SVM in order to perform the classification task. Pain in this specific work is elicited throughout tonic cold. Most recently, Thiam et al. [31,32] provided the results for a row of pain intensity classification experiments based on the SenseEmotion Database (SEDB) [33], by using several fusion architectures to merge hand-crafted features extracted from different modalities, including physiological, audio and video channels. Thereby, the combination of the features extracted from the recorded signals was compared for different fusion approaches, namely the fusion at feature level, the fusion at the classifiers' output level and the fusion at an intermediate level. Random Forests [34] were used as the base classifiers. In [35], the authors combined camera PPG input signals with ECG and EMG signals in order to proceed with a user-independent pain intensity classification using the same dataset. The authors used a fusion architecture at the feature level with Random Forests and SVMs as base classifiers.

In [36-38], the authors performed different pain intensity classification experiments based on the BioVid Heat Pain Database [39] (BVDB). All the conducted experiments were based on a carefully selected set of features extracted from both physiological and video channels. The classification was also performed using either Random Forests or SVMs. In [40], Kächele et al. performed a user-independent pain intensity classification evaluation based on physiological input signals, using the same dataset. The authors used the whole data from all recorded pain levels in a classification, as well as a regression setting with Random Forests as the base classifiers. Several personalisation techniques were designed and validated, based on meta information from the test subjects, distance measures and machine learning techniques. The same authors proposed an adaptive confidence learning approach for personalised pain estimation in [41] based on both physiological and video modalities. Thereby, the authors applied the fusion at feature level. The whole pain intensity estimation task was analysed as a regression problem. Random Forests were used as the base regression models. Moreover, a multi-layer perceptron (MLP) was applied to compute the confidence for an additional personalisation step. One recent work included the physiological signals of both datasets (SEDB and BVDB) [42]. The authors analysed different fusion approaches with fixed aggregating rules based on their merging level for the person-independent multi-class scenario using all available pain levels. Thereby, three of the most popular decision tree based classifier systems, i.e., Bagging [43], Boosting [44] and Random Forests, were compared.

The current work focuses on the application of deep learning approaches for nociceptive heat-induced pain recognition based on physiological signals (EMG, ECG and electrodermal activity (EDA)). Several deep learning architectures are proposed for the assessment of measurable physiological parameters in order to perform an end-to-end classification of different levels of artificially induced nociceptive pain. The current work aims at achieving state-of-the-art classification performances based on feature learning (the designed architecture autonomously extracts relevant features from the preprocessed raw signals in an end-to-end manner), therefore removing the reliance on expert knowledge for the design and optimisation of reliable pain intensity classification models (since most of the previous works on pain intensity classification involving autonomic parameters rely on a carefully designed set of hand-crafted features). The remainder of the work is organised as follows. The proposed deep learning approaches as well as the dataset used for the validation of the approaches are described in Section 2. Subsequently, 
a description of the results corresponding to the conducted assessments specific to each presented approach is provided in Section 3. Finally, the findings of the conducted experiments are discussed in Section 4, followed by the description of potential future works and a conclusion.

\section{Materials and Methods}

\subsection{BioVid Heat Pain Database (BVDB)}

The BioVid Heat Pain Database [39] (BVDB) was collected at Ulm University. It includes multi modal data recordings from healthy subjects subjected to different levels of artificially induced pain stimuli under strictly controlled conditions. The pain elicitation in the form of heat was conducted through the professionally designed PATHWAY (http:/ / www.medoc-web/products / pathway) thermode attached to the participants' right forearm. Before the data were recorded, a personalised calibration step was undertaken for each participant to determine individual levels for the pain threshold, as well as the tolerance threshold. Therefore, starting at a temperature of $32{ }^{\circ} \mathrm{C}$ (global pain free level $T_{0}$ for all participants), the temperature was slowly increased until, first, the participant felt a change from heat to pain (pain threshold $T_{1}$ ), and, second, the pain became hardly bearable (tolerance threshold $T_{4}$ ). In addition, two in-between pain elicitation levels $T_{2}$ and $T_{3}$ were calculated, making the four individual pain levels $T_{1}, T_{2}, T_{3}, T_{4}$ equidistant. After the initial calibration steps, starting at the baseline temperature $T_{0}$, each of the four individual pain levels was applied randomly 20 times. Each of the pain levels was held for a total of $4 \mathrm{~s}$. Each pain stimulation was followed by a rest period during which the baseline temperature was held for a random duration of 8-12 s. Ninety subjects were recruited for the experiments. The participants covered three age groups, i.e., 18-35 years, 36-50 years and 51-65 years. Each group was equally distributed, including 15 male and 15 female subjects. In the current study, the designed approaches were assessed on the BioVid Heat Pain Database (Part A) since most of the related works were conducted based on this specific database. The database is publicly available and consists of a total of 87 participants. Due to technical issues during the recordings, some of the data specific to three participants are missing [36]. Those participants were therefore discarded and the remaining 87 participants, for which all data are available, constitute the BioVid Heat Pain Database (Part A).

During the experiments, three different physiological signals were recorded, namely electrodermal activity (EDA), electrocardiogram (ECG) and electromyogram (EMG) (a sample of the recorded physiological signals is depicted in Figure 1). The EDA is an indicator of the skin conductance level and was measured at both, the participants' index and ring fingers. The ECG signals measure the participants' heart activity, such as the heart rate, the interbeat interval and the heart rate variability. The EMG signal is an indicator of the muscle activity. The EMG signal of the current dataset consists of the muscle activities of the trapezius muscles, which are located at the back, in the shoulder area. In addition to the biopotentials, different video signals were recorded. Since in the current work we only consider the physiological signals, interested readers are referred to [39] to get further details on the whole dataset. Having 20 elicitations for each level of pain elicitation, every subject is represented by a total of $20 \times 5=100$ sequences of numerical data points (time series). Therefore, the unprocessed dataset consists of $87 \times 100=8700$ samples, each labelled with its corresponding level of nociceptive pain elicitation $\left(T_{0}\right.$, $T_{1}, T_{2}, T_{3}$ or $\left.T_{4}\right)$. 


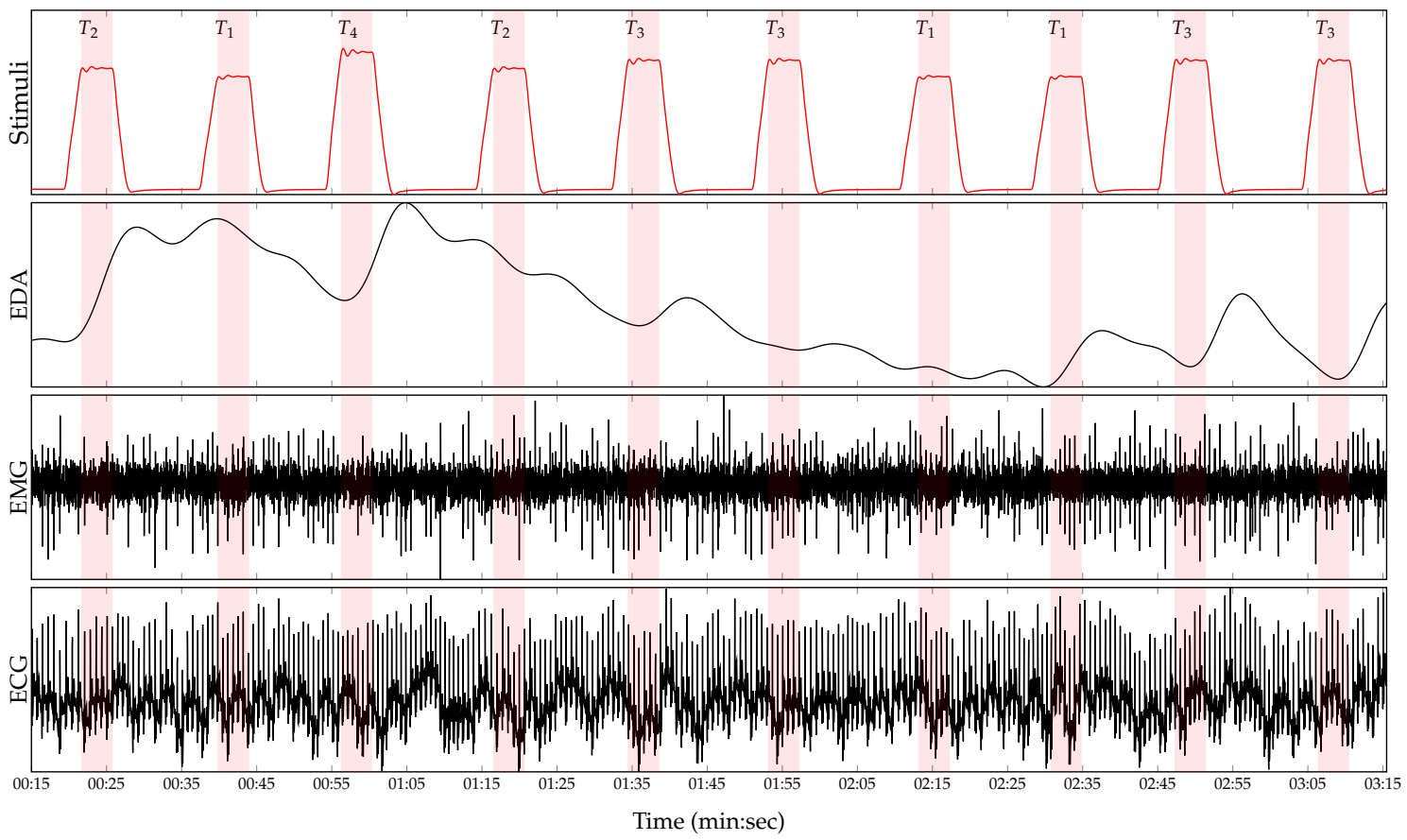

Figure 1. Recorded physiological data. From top to bottom: Series of artificially induced pain elicitation ( $T_{1}$, pain threshold temperature; $T_{2}$, first intermediate elicitation temperature; $T_{3}$, second intermediate elicitation temperature; $T_{4}$, pain tolerance temperature); $\operatorname{EDA}(\mu \mathrm{S})$; $\mathrm{EMG}(\mu \mathrm{V})$; and $\mathrm{ECG}(\mu \mathrm{V})$.

\subsection{Data Preprocessing}

Prior to the classification experiments, the sampling rate of the recorded physiological modalities was reduced to $256 \mathrm{~Hz}$, in order to reduce the computational requirements. Subsequently, the amount of noise and artefacts within the recorded data was significantly reduced by applying different signal preprocessing techniques on each specific modality. A third-order low-pass Butterworth filter with a cut-off frequency of $0.2 \mathrm{~Hz}$ was applied on the EDA signals. The EMG signals were filtered by applying a fourth-order bandpass Butterworth filter with a frequency range of $[20,250] \mathrm{Hz}$. Finally, a third-order bandpass Butterworth filter with a frequency range of $[0.1,250] \mathrm{Hz}$ was applied on the ECG signals. Furthermore, the data were segmented as proposed in [37], but rather than using $5.5 \mathrm{~s}$ windows with a shift of $3 \mathrm{~s}$ from the elicitations' onset, the preprocessed signals were segmented into windows of length $4.5 \mathrm{~s}$, with a shift from the elicitations' onset of $4 \mathrm{~s}$ (see Figure 2a), as recently proposed in [31]. Each signal extracted within this window constitutes a 1D array of size $4.5 \times 256=1152$ and was later used in combination with the corresponding level of nociceptive pain elicitation to optimise and assess the designed deep classification architectures. Thus, each physiological modality specific to each single participant is represented by a tensor with the dimensionality $100 \times 1152 \times 1$. After some close analysis of the preprocessed physiological signals, a clear baseline wandering of the ECG signal, which is characterised by a strong correlation with the shape of the EDA signal, was observed (see Figure 2b). Therefore, the segmented ECG signals were additionally detrended by subtracting a fifth-degree polynomial least-squares fit from the filtered signals. This step was carried out to remove the aforementioned artefacts from the ECG signals, since these artefacts could potentially bias the classification performance of the corresponding deep classification model (instead of using information stemming uniquely from the ECG signal, the designed system would end up extracting information stemming from a non-linear combination of both the ECG signal and a noisy signal related to the EDA signal). Finally, data augmentation was performed by shifting the $4.5 \mathrm{~s}$ window of segmentation backward and forward in time with small shifts of length $250 \mathrm{~ms}$ and a maximal 
total window shift of $1 \mathrm{~s}$ in each direction, starting from the initial position of the window depicted in Figure 2a. The signals extracted within these windows were subsequently used as training material for the optimisation of the classification architectures.

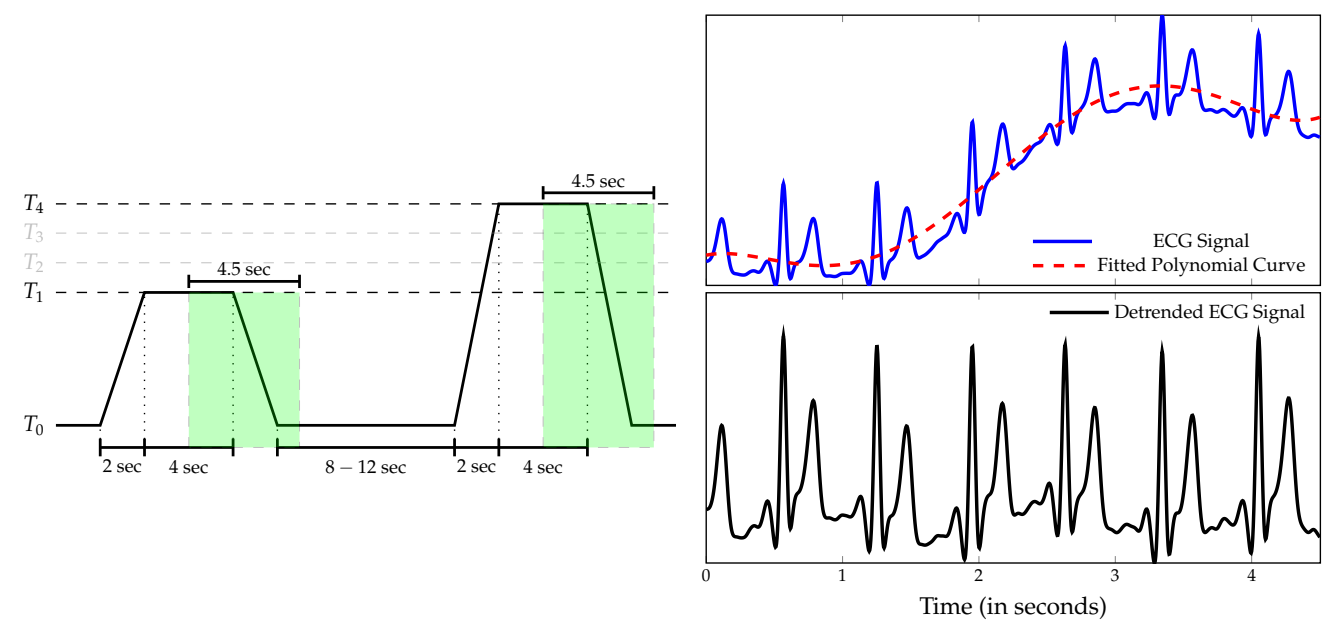

(a)

(b)

Figure 2. Data preprocessing. (a) Signal Segmentation. The classification experiments were performed on windows of length $4.5 \mathrm{~s}$ with a temporal shift of $4 \mathrm{~s}$ from the elicitations' onset. (b) The ECG signal was further detrended by subtracting a least-squares polynomial fit from the preprocessed signal.

\subsection{Uni-modal Deep Model Description}

As mentioned above, the goal of the current work is to apply feature learning to alleviate the reliance on domain specific expert knowledge that occurs when relevant and adequate features are to be manually designed (hand-crafted features) in order to achieve state-of-the-art classification performances. Therefore, multi-layer CNNs were designed and fed with the preprocessed physiological signals in order to automatically compute relevant signal representations and at the same time optimise the classification architectures. In the following sections, $c$ depicts the number of classes of the classification task.

CNNs $[45,46]$ constitute a distinct category of biologically inspired neural networks, which are characterised by a hierarchical structure of several processing layers. The input to a CNN is sequentially and progressively transformed by each specific layer and the back-propagated information stemming from the error computed between the network's output and the expected output (ground-truth) is used to optimise the whole structure of the architecture in order to efficiently and effectively solve a classification or regression task. The basic processing layers of CNNs are convolutional layers, pooling layers and fully connected layers. Convolutional layers are characterised by a set of neurons (or kernels), whereby each specific neuron extracts a specific pattern of information from a patch of the layer's input. Each neuron consists of a set of trainable weights, the size of which is determined by the patch's size (or kernel size). The output of each neuron is calculated by applying a non-linear activation function (e.g., sigmoid function) on the weighted sum of the neuron's input. Each neuron scans the layer's input sequentially and the aggregation of the resulting local information extracted at each specific patch constitutes a feature map. Thus, the output of a convolutional layer is a set of feature maps generated by the convolution of each neuron across the layer's input. Pooling layers reduce the spatial resolution of the generated feature maps by merging semantically similar features. Max Pooling is a commonly used pooling approach and consists of computing the maximum value of a defined local patch (the size of the patch related to a specific pooling layer is referred in the current work as "pool size") of each feature map. Fully connected layers are basically 
single-layer feed-forward networks that perform the classification or regression task based on the learned deep representations.

Several challenges emerge when it comes to optimising such architectures. One of those challenges is the so-called vanishing or exploding gradients problem, which is caused by the internal covariate shift (constant fluctuations in layers' input distributions) occurring in deep architectures during the training process. In [47], the authors proposed a technique called Batch Normalisation to address this specific issue. Batch Normalisation consists of automatically learning the optimal scaling and shifting parameters of each layer's input, so that each layer's input is dynamically normalised, thus significantly reducing the effects of the internal covariate shift and therefore stabilising the training process. Another common challenge occurring when training CNNs is the overfitting problem caused by the large amount of parameters that have to be consistently and effectively optimised. Applying regularisation techniques can help to significantly reduce this issue. The authors of [48] introduced the dropout approach, which is one of the most commonly used regularisation techniques for deep neural networks. The dropout approach consists of randomly and temporarily removing a set of neurons (or units) from the neural network during each training step, each neuron having a fixed probability $p \in[0,1]$ of being retained. The resulting model is therefore more robust against overfitting and generalises better.

In the current work, the designed architectures are regularised using both techniques and the dropout rate is fixed at 25\%. Moreover, the Exponential Linear Unit (ELU) function [49] defined in Equation (1)

$$
e^{e l u_{\alpha}}(x)= \begin{cases}\alpha(\exp (x)-1) & \text { if } x<0 \\ x & \text { if } x \geq 0\end{cases}
$$

is used as activation function for both convolutional layers and fully connected layers (with $\alpha=1$ ), except for the last fully connected layer of each architecture where a softmax function defined in Equation (2)

$$
s\left(y_{i}\right)=\frac{\exp \left(y_{i}\right)}{\sum_{j} \exp \left(y_{j}\right)}
$$

is used as activation function, where $y_{i}=e l u_{\alpha}\left(\sum_{k=1}^{n} w_{i, k} x_{k}+b_{i}\right)\left(\left\{w_{i, k}\right\}_{k=1}^{n}\right.$ represents the set of weights of the $i$ th neuron, $b_{i}$ represents the bias term of the $i$ th neuron and $x=\left(x_{1}, \ldots, x_{k}, \ldots, x_{n}\right)$ represents the output of the precedent fully connected layer). The designed architectures for each physiological signal are based on 1D convolutional layers and are described in Table 1. The architectures are similar and were inspired by the architecture presented in [50] for the classification of ECG signals. The unique difference between the architectures is the usage of a dropout layer after each convolutional layer in the architecture specific to both modalities EMG and ECG. 
Table 1. Deep classification architectures for each of the recorded physiological modality.

\begin{tabular}{|c|c|c|c|c|c|c|c|}
\hline \multicolumn{4}{|c|}{ EDA } & \multicolumn{4}{|c|}{ EMG \& ECG } \\
\hline Layer Name & No. Kernels (Units) & Kernel (Pool) Size & Stride & Layer Name & No. Kernels (Units) & Kernel (Pool) Size & Stride \\
\hline Convolution & 16 & 3 & 1 & Convolution & 16 & 11 & 1 \\
\hline Batch Normalisation & - & - & - & Batch Normalisation & - & - & - \\
\hline Max Pooling & - & 2 & 2 & Max Pooling & - & 2 & 2 \\
\hline Convolution & 16 & 3 & 1 & Dropout & - & - & - \\
\hline Batch Normalisation & - & - & - & Convolution & 16 & 11 & 1 \\
\hline Max Pooling & - & 2 & 2 & Batch Normalisation & - & - & - \\
\hline Convolution & 32 & 3 & 1 & Max Pooling & - & 2 & 2 \\
\hline Batch Normalisation & - & - & - & Dropout & - & - & - \\
\hline Max Pooling & - & 2 & 2 & Convolution & 32 & 11 & 1 \\
\hline Convolution & 32 & 3 & 1 & Batch Normalisation & - & - & - \\
\hline Batch Normalisation & - & - & - & Max Pooling & - & 2 & 2 \\
\hline Max Pooling & - & 2 & 2 & Dropout & - & - & - \\
\hline Convolution & 64 & 3 & 1 & Convolution & 32 & 11 & 1 \\
\hline Batch Normalisation & - & - & - & Batch Normalisation & - & - & - \\
\hline Max Pooling & - & 2 & 2 & Max Pooling & - & 2 & 2 \\
\hline Convolution & 64 & 3 & 1 & Dropout & - & - & - \\
\hline Batch Normalisation & - & - & - & Convolution & 64 & 11 & 1 \\
\hline Max Pooling & - & 2 & 2 & Batch Normalisation & - & - & - \\
\hline Convolution & 128 & 3 & 1 & Max Pooling & - & 2 & 2 \\
\hline Batch Normalisation & - & - & - & Dropout & - & - & - \\
\hline Max Pooling & - & 2 & 2 & Convolution & 64 & 11 & 1 \\
\hline Flatten & - & - & - & Batch Normalisation & - & - & - \\
\hline Fully Connected & 1024 & - & - & Max Pooling & - & 2 & 2 \\
\hline Dropout & - & - & - & Dropout & - & - & - \\
\hline Fully Connected & 512 & - & - & Convolution & 128 & 11 & 1 \\
\hline Dropout & - & - & - & Batch Normalisation & - & - & - \\
\hline \multirow[t]{8}{*}{ Fully Connected } & c & - & - & Max Pooling & - & 2 & 2 \\
\hline & & & & Flatten & - & - & - \\
\hline & & & & Dropout & - & - & - \\
\hline & & & & Fully Connected & 1024 & - & - \\
\hline & & & & Dropout & - & - & - \\
\hline & & & & Fully Connected & 512 & - & - \\
\hline & & & & Dropout & - & - & - \\
\hline & & & & Fully Connected & c & - & - \\
\hline
\end{tabular}

ELU is used as activation function in both convolutional and fully connected layers, except for the last fully connected layer where a softmax activation function is used. The networks are further regularised by using dropout layers with a fixed dropout rate of $25 \%$.

\subsection{Multi-Modal Deep Model Description}

To further investigate the compatibility of the recorded physiological data, several fusion approaches based on CNNs are proposed. The information stemming from each modality is aggregated at different levels of abstraction.

The first approach depicted in Figure 3 consists of an early fusion method, where the aggregation is done at the lowest level of abstraction, which consists of the preprocessed raw signals (input data). A 2D representation of the input data is generated by concatenating the three physiological modalities along the temporal axis, resulting in a tensor with the dimensionality $3 \times 1152 \times 1$. The resulting data are subsequently fed into a network consisting of $2 \mathrm{D}$ convolutional layers. The motivation behind such an approach is to enable the architecture to dynamically learn an appropriate set of weights, which will generate feature maps consisting of relevant and compatible information extracted simultaneously from 
the recorded modalities, when applied to the 2D data representation. The designed fusion architecture is described in Table 2.

Table 2. Early fusion deep CNN architecture.

\begin{tabular}{|c|c|c|c|}
\hline Layer Name & No. Kernels (Units) & Kernel (Pool) Size & Stride \\
\hline Convolution & 16 & $2 \times 11$ & $1 \times 1$ \\
\hline Convolution & 16 & $2 \times 11$ & $1 \times 1$ \\
\hline Batch Normalisation & - & - & - \\
\hline Max Pooling & - & $1 \times 2$ & $1 \times 2$ \\
\hline Dropout & - & - & - \\
\hline Convolution & 32 & $1 \times 11$ & $1 \times 1$ \\
\hline Batch Normalisation & - & - & - \\
\hline Max Pooling & - & $1 \times 2$ & $1 \times 2$ \\
\hline Dropout & - & - & - \\
\hline Convolution & 32 & $1 \times 11$ & $1 \times 1$ \\
\hline Batch Normalisation & - & - & - \\
\hline Max Pooling & - & $1 \times 2$ & $1 \times 2$ \\
\hline Dropout & - & - & - \\
\hline Convolution & 64 & $1 \times 11$ & $1 \times 1$ \\
\hline Batch Normalisation & - & - & - \\
\hline Max Pooling & - & $1 \times 2$ & $1 \times 2$ \\
\hline Dropout & - & - & - \\
\hline Convolution & 64 & $1 \times 11$ & $1 \times 1$ \\
\hline Batch Normalisation & - & - & - \\
\hline Max Pooling & - & $1 \times 2$ & $1 \times 2$ \\
\hline Flatten & - & - & - \\
\hline Dropout & - & - & - \\
\hline Fully Connected & 1024 & - & - \\
\hline Dropout & - & - & - \\
\hline Fully Connected & 512 & - & - \\
\hline Dropout & - & - & - \\
\hline Fully Connected & c & - & - \\
\hline
\end{tabular}

The architecture is based on 2D convolutional layers. A 2D representation of the input data is generated by concatenating the three physiological modalities resulting in a tensor with the dimensionality $3 \times 1152 \times 1$. Similar to the previous architectures (see Table 1), ELU is used as activation function in both convolutional and fully connected layers, except for the last fully connected layer where a softmax activation function is used. The network is further regularised by using dropout layers with a fixed dropout rate of $25 \%$. 

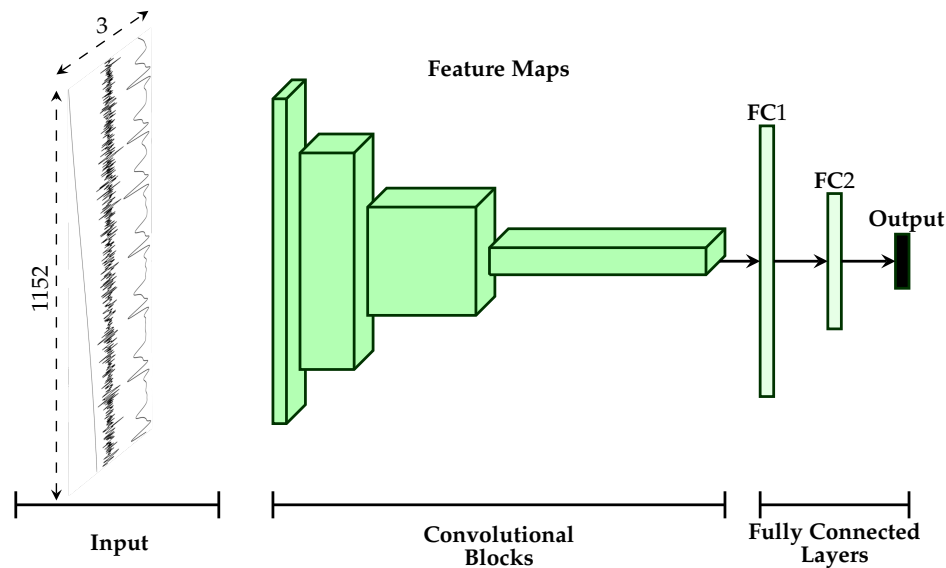

Figure 3. Early Fusion Architecture. A 2D representation of the input data is generated by concatenating the three physiological modalities and is subsequently fed into the designed deep architecture.

Furthermore, two additional late fusion approaches are proposed (see Figure 4). Both approaches are based on the uni-modal CNN architectures described earlier (see Section 2.3). The first approach described in Figure 4a performs the aggregation of the information at the mid-level since it involves using intermediate representations of the input data. It consists of concatenating the outputs of the second fully connected layer of each modality specific architecture and feeding the resulting representation to an output layer with a softmax activation function. The second approach depicted in Figure $4 \mathrm{~b}$ performs the aggregation at the highest level of abstraction, since it involves using the respective softmax layers' outputs of each modality specific architecture. An additional layer consisting of a set of trainable positive parameters $\left(\alpha_{1}, \alpha_{2}, \alpha_{3}\right) \in \mathbb{R}_{\geq 0}^{3}$ with a linear activation function is directly connected to the outputs of each uni-modal architecture.

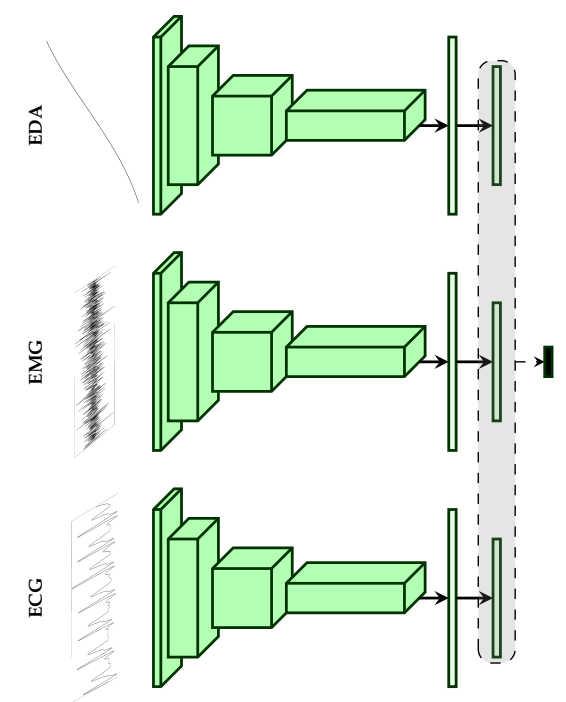

(a)

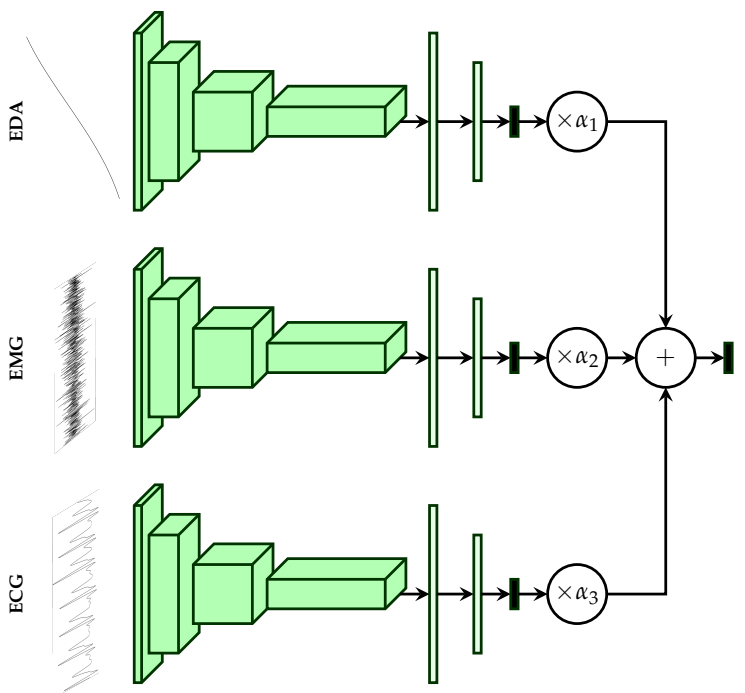

(b)

Figure 4. Late Fusion Architectures. (a) The features extracted by the second fully connected layer are concatenated and fed into the output layer. (b) The final output consists of a weighted average of the outputs of each uni-modal model. 
For each modality specific architecture $i \in\{1,2,3\}$ (since we are dealing with three physiological modalities), let $\left\{\theta_{i, j} \in[0,1]: 1 \leq j \leq \mathrm{c}\right\}$ be the output values of the respective softmax layers. The output of the aggregation layer is computed by using the following formulas:

$$
\begin{gathered}
e_{j}=\frac{1}{3}\left(\sum_{i=1}^{3} \alpha_{i} \theta_{i, j}\right), \text { with the constraint: } \sum_{i=1}^{3} \alpha_{i}=1 \\
s\left(e_{j}\right)=e_{j}
\end{gathered}
$$

First, a weighted average output of the class probabilities stemming from the uni-modal architectures is computed (see Equation (3)), and the corresponding class probabilities of the fusion architecture are subsequently deducted by applying a linear activation function on the previously computed scores (see Equation (4)). Furthermore, the whole architecture is trained by using the loss function defined in Equation (5),

$$
L=\sum_{i=1}^{3} \lambda_{i} L_{i}+\lambda_{a g g} L_{a g g}
$$

where $L_{1}, L_{2}$ and $L_{3}$ are the loss functions of each modality specific architecture and $L_{a g g}$ is the loss function of the aggregation layer. The parameters $\lambda_{1}, \lambda_{2}, \lambda_{3}$ and $\lambda_{\text {agg }}$ are the corresponding weights for each of the loss functions. Once the architecture has been trained, unseen samples are classified based uniquely on the output of the aggregation layer. All described fusion approaches are subsequently trained in an end-to-end manner, which means that the fusion parameters are optimised at the same time as the parameters of each modality specific classification architecture. Furthermore, the parameters of each described architecture (uni-modal as well as multi-modal) are optimised using the cross entropy loss function defined in Equation (6),

$$
\text { Loss }=-\sum_{j=1}^{c} y_{j} \log \left(\hat{y}_{j}\right)
$$

where $y_{j}$ is the ground-truth value of the $j$ th class and $\hat{y}_{j}$ is the $j$ th output value of the softmax function. Concerning the second late fusion architecture, the cross entropy loss function is used for each uni-modal architecture as well as for the aggregation layer $\left(L_{1}=L_{2}=L_{3}=L_{\text {agg }}=\right.$ Loss $)$.

\section{Results}

All previously described deep architectures are trained using the Adaptive Moment estimation (Adam) [51] optimisation algorithm with a fixed learning rate set empirically to $10^{-5}$. The training process consisted of 100 epochs with the batch size set to 100. The weights of the loss function for the second late fusion architecture (see Figure $4 \mathrm{~b}$ ) were empirically set as follows: $\lambda_{1}=\lambda_{2}=\lambda_{3}=0.2, \lambda_{\text {agg }}=0.4$. The weight corresponding to the aggregation layer $\left(\lambda_{\text {agg }}\right)$ was set higher than the others to push the network to focus on the weighted combination of the single modality architectures' outputs, and therefore to evaluate an optimal set of the weighting parameters $\left\{\alpha_{1}(E D A), \alpha_{2}(E M G), \alpha_{3}(E C G)\right\}$. The implementation and evaluation of the described algorithms was done with the libraries Keras [52], Tensorflow [53] and Scikit-learn [54]. The evaluation of the architectures was performed in a Leave-One-Subject-Out (LOSO) cross-validation setting, which means that 87 experiments were conducted. During each experiment, the data specific to a single participant were used to evaluate the performance of the trained deep model and were never seen during the optimisation of this specific deep model. The data specific to each single 
participant were therefore used once as an unseen test set, and the results depicted in this section consist of averaged performance metrics from a set of 87 performance values.

A performance evaluation of the designed architectures in a binary classification task consisting of the discrimination between the baseline temperature $T_{0}$ and the pain tolerance temperature $T_{4}$ is reported in Table 3. The achieved results based on CNNs are also compared to the state-of-the-art results reported in previous works. At a glance, the designed deep learning architectures outperform the state-of-the-art results in every setting, except for the ECG modality. Regarding the aggregation of all physiological modalities, the second late fusion architecture performs best and sets a new state-of-the-art fusion performance with an average accuracy of $84.40 \%$, which even outperforms the best fusion results reported in [41], where the authors could achieve an average classification performance of $83.1 \%$ by using both physiological and video features.

Table 3. Performance comparison to early work on the BVDB (Part A) for the classification task $T_{0}$ vs. $T_{4}$ in a LOSO cross-validation setting.

\begin{tabular}{ccccc}
\hline Method & ECG & EMG & EDA & Fusion \\
\hline Werner et al. [36] & $\mathbf{6 2 . 0 0}$ & 57.90 & 73.80 & 74.10 \\
\hline Kächele et al. [40,41] & 53.90 & 58.51 & 81.10 & 82.73 \\
\hline & & & & Early Fusion: $82.79 \pm 15.22$ \\
Our Approaches (CNNs) & $57.04 \pm 11.58$ & $\mathbf{5 8 . 6 5} \pm \mathbf{1 3 . 8 2}$ & $\mathbf{8 4 . 5 7} \pm \mathbf{1 4 . 1 3}$ & Late Fusion (a): $83.39 \pm 15.54$ \\
\end{tabular}

The performance metric consists of the average accuracy (in \%) over the LOSO cross-validation evaluation (the standard deviation of the cross-validation results for the proposed approaches is also provided). The best performing approach for each modality and the aggregation of all modalities is depicted in bold.

The deep architecture based on the EDA modality significantly outperforms all previously reported classification results with an average accuracy of $84.57 \%$.

Based on these findings, further classification experiments were conducted, based on each physiological modality and also the best performing fusion architecture (Late Fusion (b)). The performance evaluation of the conducted experiments consisting of several binary classification experiments and a multi-class classification experiment is summarised in Table 4.

EDA significantly outperforms both EMG and ECG in all conducted classification experiments and constitutes the best performing single modality, which is consistent with the results reported in previous works. Both EMG and ECG depict similar classification performances and also perform poorly for almost all classification experiments. The discrimination between the baseline temperature $T_{0}$ and the pain threshold temperature $T_{1}$, as well as the two intermediate temperatures $T_{2}$ and $T_{3}$, constitute very difficult classification experiments that both modalities are unable to perform successfully. However, the classification performances of both modalities for the classification tasks $T_{0}$ vs. $T_{4}$ and $T_{1}$ vs. $T_{4}$ are significantly above chance level, which shows that higher temperatures of elicitation cause observable and measurable responses in the recorded physiological signals, that can be used to perform the classification tasks at a certain degree of satisfaction. However, the overall performance of the fusion architecture is greatly affected by the significantly poor performance of both ECG and EMG in comparison to EDA. As can be seen in Table 4, the EDA classification architecture outperforms the fusion architecture in almost all classification experiments (but not significantly), except for the classification task $T_{1}$ vs. $T_{4}$ and the multi-class classification task (the performance improvement of the fusion architecture is however not significant). 
Table 4. CNN Classification performance on the BVDB (Part A) in a LOSO cross-validation setting (the multi-class classification task corresponds to the five-class classification task $T_{0}$ vs. $T_{1}$ vs. $T_{2}$ vs. $T_{3}$ vs. $T_{4}$ ).

\begin{tabular}{|c|c|c|c|c|}
\hline Task & ECG & EMG & EDA & Late Fusion (b) \\
\hline$T_{0}$ vs. $T_{1}$ & $49.71 \pm 06.90$ & $49.71 \pm 02.77$ & $61.67 \pm 12.54^{\dagger}$ & $61.15 \pm 12.22^{\mathrm{a}, \mathrm{b}}$ \\
\hline$T_{0}$ vs. $T_{2}$ & $50.72 \pm 07.30$ & $50.29 \pm 03.60$ & $66.93 \pm 16.19^{\dagger}$ & $66.81 \pm 15.92^{a, b}$ \\
\hline$T_{0}$ vs. $T_{3}$ & $52.87 \pm 09.32$ & $53.25 \pm 08.93$ & $76.38 \pm 14.70^{\dagger}$ & $76.29 \pm 14.62^{a, b}$ \\
\hline$T_{0}$ vs. $T_{4}$ & $57.04 \pm 11.58$ & $58.65 \pm 13.82$ & $84.57 \pm 14.13^{\dagger}$ & $84.40 \pm 14.43^{\mathrm{a}, \mathrm{b}}$ \\
\hline$T_{1}$ vs. $T_{4}$ & $58.07 \pm 12.36$ & $58.79 \pm 12.08$ & $76.61 \pm 15.38$ & $76.72 \pm 15.02^{a, b, t}$ \\
\hline Multi-Class & $23.23 \pm 05.62$ & $22.85 \pm 05.65$ & $36.25 \pm 09.01$ & $36.54 \pm 08.55^{a, b, \dagger}$ \\
\hline
\end{tabular}

The performance metric consists of the average accuracy (in \%) over the LOSO cross-validation evaluation (the standard deviation of the cross-validation results is also provided). The best performing approach for each classification task is depicted in bold. We also performed a significance test between the fusion approach and each single modality, using a Wilcoxon signed rank test with a significance level of $5 \%$ : $\left({ }^{\mathrm{a}}\right)$ indicates a significant performance improvement between EMG and the fusion approach; $\left(^{b}\right)$ indicates a significant performance improvement between ECG and the fusion approach; and $\left(^{+}\right)$indicates no significant improvement between EDA and the fusion approach.

The information stemming from both modalities EMG and ECG harms the optimisation process of the fusion architecture due to its inconsistency. However, it can be seen in Figure 5 that the fusion architecture is able to detect the sources of inconsistent information and dynamically adapt by systematically assigning higher weight values to EDA, while both ECG and EMG are assigned significantly lower weight values for all conducted classification tasks, and therefore improving the generalisation ability of the fusion architecture.

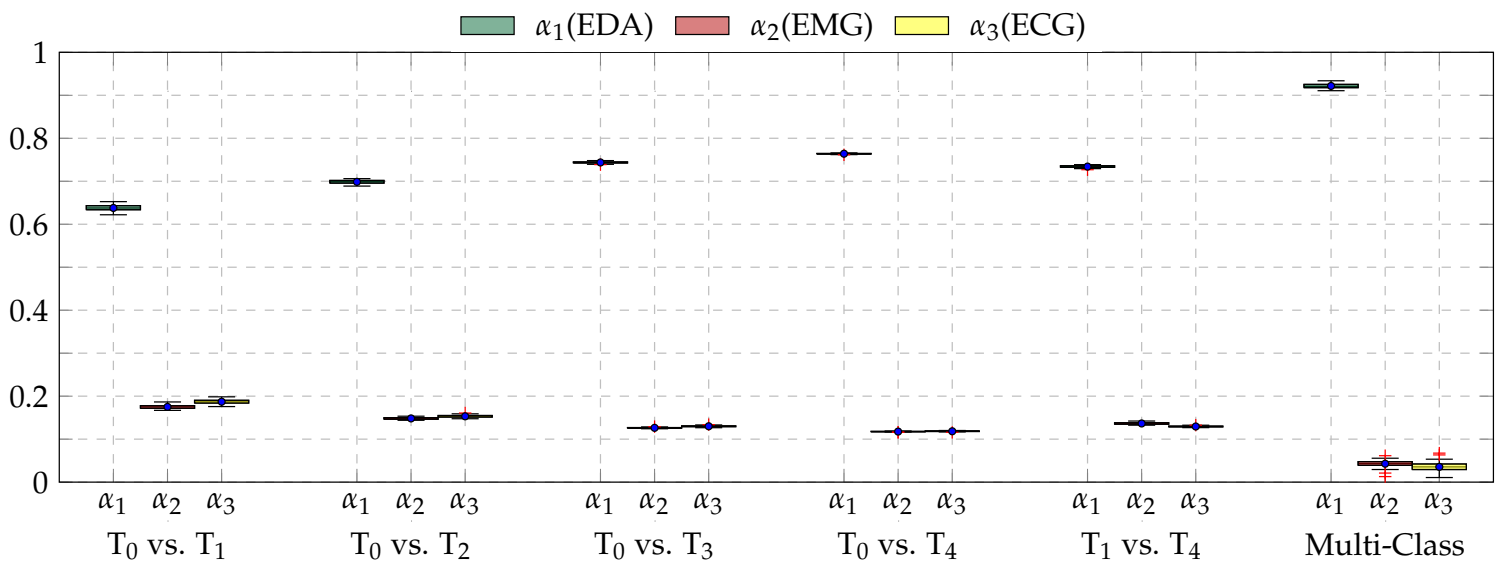

Figure 5. Box plots of the weighting parameters $\alpha_{1}, \alpha_{2}$ and $\alpha_{3}$ for the late fusion architecture (Late Fusion (b)), computed during the LOSO cross-validation evaluation of each conducted classification experiment. Within each box plot, the mean and median values of the performed LOSO cross-validation evaluation are depicted with a dot and a horizontal line, respectively.

Subsequently, the performance of both EDA and late fusion architectures were further evaluated using different performance measures. In the case of binary classification experiments, true positives $(t p)$ correspond to the number of correct acceptances, false positives $(f p)$ correspond to the number of false acceptances, true negatives $(t n)$ correspond to the number of correct rejections and false negatives ( $f n)$ correspond to the number of false rejections. These four values stem from the confusion matrix of an 
evaluated inference model and are used to define different performance measures. Those used for the current evaluation of the designed classification architectures are defined in Table 5.

The performance evaluation of the EDA architecture is depicted in Figure 6, while the performance evaluation of the fusion architecture is depicted in Figure 7. Considering binary classification experiments, both architectures are able to consistently discriminate between the baseline temperature $T_{0}$ and the other temperatures of pain elicitation. However, the performance of both architectures with regards to the five-class classification experiment suggests that the discrimination between all five levels of pain elicitation is a very challenging classification task. While the overall accuracy of each architecture is significantly above random performance (which is $20 \%$ in the case of a five-class classification task), the discrimination of the intermediate levels of pain elicitation remains very difficult, as can be seen in Figure 8 . Both baseline and pain tolerance temperatures $T_{0}$ and $T_{4}$ can be classified with a relatively good performance. The classification performance of $T_{2}$ is barely above random performance and both $T_{1}$ and $T_{3}$ are mostly confused with $T_{0}$ and $T_{4}$, respectively. These results are however consistent with previous works on the same dataset.

Table 5. Classification performance measures.

\begin{tabular}{lcc}
\hline Measure & Binary Classification & Multi-Class Classification \\
\hline Accuracy & $\frac{t p+t n}{t p+t n+f p+f n}$ & $\frac{1}{c} \sum_{i=1}^{c} \frac{t p_{i}+t n_{i}}{t p_{i}+t n_{i}+f p_{i}+f n_{i}}$ \\
\hline Precision & $\frac{t p}{t p+f p}$ & $\frac{1}{c} \sum_{i=1}^{c} \frac{t p_{i}}{t p_{i}+f p_{i}}$ \\
\hline Recall & $\frac{t p}{t p+f n}$ & $\frac{1}{c} \sum_{i=1}^{c} \frac{t p_{i}}{t p_{i}+f n_{i}}$ \\
\hline F1 score & $\frac{2 \times \text { Precision } \times \text { Recall }}{\text { Precison }+ \text { Recall }}$ \\
\hline
\end{tabular}

In the case of multi-class classification experiments: $t p_{i}$ corresponds to true positives, $t n_{i}$ corresponds to true negatives, $f p_{i}$ corresponds to false positives and $f n_{i}$ corresponds to false negatives in the confusion matrix associated with the $i$ th class. Furthermore, since the dataset used for the evaluation of the performance of the designed architectures is balanced, we use the macro-averaged F1 score in the case of multi-class classification.

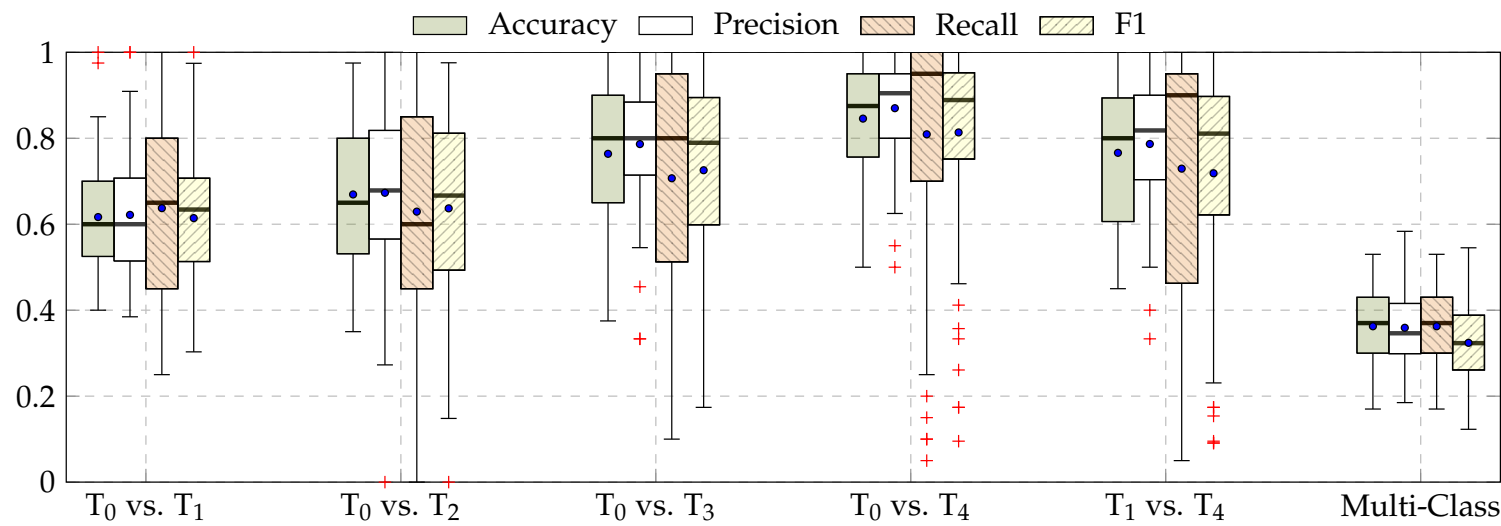

Figure 6. EDA classification performance. Within each box plot, the mean and median values of the respective performance evaluation metrics are depicted with a dot and a horizontal line, respectively. 


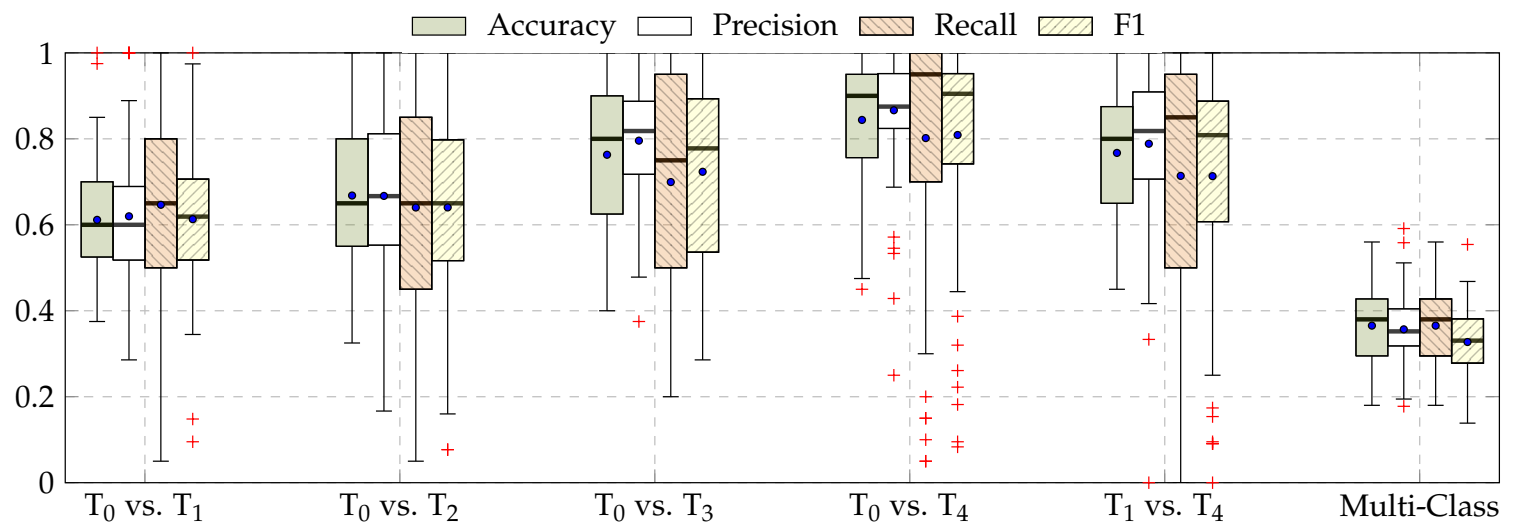

Figure 7. Late fusion classification performance (Late Fusion (b)). Within each box plot, the mean and median values of the respective performance evaluation metrics are depicted with a dot and a horizontal line, respectively.

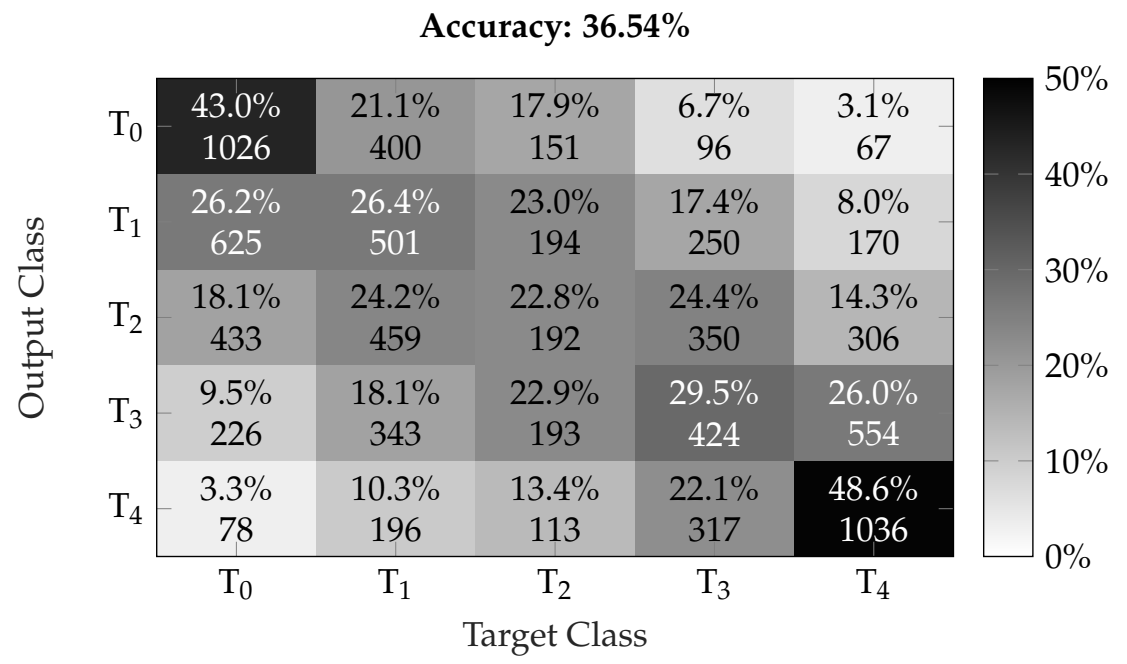

Figure 8. Multi-class classification performance (confusion matrix) of the fusion architecture (Late Fusion (b)). The darker the color the higher the corresponding performance.

Table 6. EDA performance comparison to early work on the BVDB (Part A) in a LOSO cross-validation setting.

\begin{tabular}{ccccc}
\hline Method & $\mathrm{T}_{\mathbf{0}}$ vs. $\mathbf{T}_{\mathbf{1}}$ & $\mathrm{T}_{\mathbf{0}}$ vs. $\mathbf{T}_{\mathbf{2}}$ & $\mathrm{T}_{\mathbf{0}}$ vs. $\mathbf{T}_{\mathbf{3}}$ & $\mathbf{T}_{\mathbf{0}}$ vs. $\mathbf{T}_{\mathbf{4}}$ \\
\hline Werner et al. [36] & 55.40 & 60.20 & 65.90 & 73.80 \\
\hline Lopez-Martinez et al. [55] & 56.44 & 59.40 & 66.00 & 74.21 \\
\hline Our Approach (CNN) & $\mathbf{6 1 . 6 7} \pm \mathbf{1 2 . 5 4}$ & $\mathbf{6 6 . 9 3} \pm \mathbf{1 6 . 1 9}$ & $\mathbf{7 6 . 3 8} \pm 14.70$ & $\mathbf{8 4 . 5 7} \pm \mathbf{1 4 . 1 3}$ \\
\hline
\end{tabular}

The performance metric consists of the average accuracy (in \%) over the LOSO cross-validation evaluation. The best performing approach for each classification task is depicted in bold. 
We therefore compared the performance of the EDA and proposed late fusion approach to early works. For the sake of fairness, we considered the related works performed on the exact same dataset, using the exact same evaluation settings (LOSO with all 87 participants). The results depicted in Table 6 clearly show that the designed CNN architecture specific to EDA is able to consistently and significantly outperform previous approaches in all binary classification settings. Moreover, the authors of $[56,57]$ reported overall accuracy performances of, respectively, $74.40 \%$ and $81.30 \%$ for the binary classification task $T_{0}$ vs. $T_{4}$ based uniquely on EDA. These approaches are also based on carefully designed hand-crafted features and are also significantly outperformed by the proposed CNN architecture specific to EDA.

Furthermore, we also compared the proposed late fusion approach with other fusion approaches proposed in early works. The results depicted in Table 7 show that the proposed fusion approach outperforms previous approaches for the binary classification task $T_{0}$ vs. $T_{4}$. Concerning the multi-class classification task, the proposed fusion approach also outperforms early approaches with an overall accuracy of $36.54 \%$. The authors of [41] reported an overall accuracy of $33 \%$ with a classification model based on physiological modalities, while Werner et al. [58] reported an overall accuracy of 30.8\% with a classification model based on head pose and facial activity descriptors.

Table 7. Fusion performance comparison to early work on the BVDB (Part A) in a LOSO cross-validation setting for the classification task $T_{0}$ vs. $T_{4}$.

\begin{tabular}{|c|c|c|}
\hline Approach & Description & Performance \\
\hline Werner et al. [58] & Early Fusion with Random Forests (Head Pose and Facial Activity Descriptors) & 72.40 \\
\hline Werner et al. [36] & Early Fusion with Random Forests (EDA, EMG, ECG, Video) & 77.80 \\
\hline Kächele et al. [56] & Early Fusion with Random Forests (EDA, ECG, Video) & 78.90 \\
\hline Kächele et al. [57] & Late Fusion with Random Forests and Pseudo-inverse (EDA, EMG, ECG, Video) & 83.10 \\
\hline Our Approach (CNN) & Late Fusion (b) with CNNs (EDA, EMG, ECG) & $84.40 \pm 14.43$ \\
\hline
\end{tabular}

The performance metric consists of the average accuracy (in \%) over the LOSO cross-validation evaluation. The best performing approach is depicted in bold.

Moreover, the designed fusion architecture was tested on the BioVid Heat Pain Database (Part $B)$. The database was generated using the same exact procedure as Part A. However, it consists of 86 participants and two additional EMG signals (from the corrugator and the zygomaticus muscles) were recorded. In this evaluation, we used the same signals as in Part A (EMG of the trapezius muscle, ECG and EDA), and used the same fusion architecture (Late fusion (b) depicted in Figure $4 b$ ). The computed results were subsequently compared with those of previous works. The corresponding results are depicted in Table 8.

Table 8. Fusion performance comparison to early work on the BVDB (Part B) in a LOSO cross-validation setting for the classification task $T_{0}$ vs. $T_{4}$.

\begin{tabular}{ccc}
\hline Approach & Description & Performance \\
\hline & Late Fusion with SVMs and Mean Aggregation (EMG (zygomaticus), & \\
Kächele et al. [56] & EMG (corrugator), EMG (trapezius), ECG, EDA, Video) & 76.60 \\
\hline & Early Fusion with SVM (EMG (zygomaticus), & 77.05 \\
\hline Walter et al. [37] & EMG (corrugator), EMG (trapezius), ECG, EDA) & $\mathbf{7 9 . 4 8} \pm \mathbf{1 4 . 9 6}$ \\
\hline Our Approach (CNN) & Late Fusion (b) with CNNs (EMG (trapezius), ECG, EDA) & .
\end{tabular}

The performance metric consists of the average accuracy (in \%) over the LOSO cross-validation evaluation. The best performing approach is depicted in bold. 
The methods reported in previous works consist of fusion approaches involving all the recorded signals and based on hand-crafted features $[37,56]$. Although the fusion approach proposed in the current work (late fusion (b)) is based only on three of the recorded physiological signals, it is still able to outperform the previously proposed approaches, as depicted in Table 8 . Therefore, it is believed that the performance of the architecture can be further improved by including the remaining signals (EMG corrugator, EMG zygomaticus, and Video) in the proposed architecture.

\section{Discussion and Conclusions}

This work explored the application of deep neural networks for pain intensity classification based on physiological data including ECG, EMG and EDA. Several CNN architectures, based on 1D and 2D convolutional layers, were designed and assessed based on the BioVid Heat Pain Database (Part A). Furthermore, several deep fusion architectures were also proposed for the aggregation of relevant information stemming from all involved physiological modalities. The proposed architecture specific to EDA significantly outperformed the results presented in previous works in all classification settings. For the classification task $T_{0}$ vs. $T_{4}$, EDA achieved a state-of-the-art average accuracy of $84.57 \%$. The proposed late fusion approach based on a weighted average of each modality specific model's output also achieved state-of-the-art performances (average accuracy of $84.40 \%$ for the classification task $T_{0}$ vs. $T_{4}$ ), but was unable to significantly outperform the deep model based uniquely on EDA.

Moreover, all designed architectures were trained in an end-to-end manner. Therefore, it is believed that the pre-training and fine tuning at different levels of abstraction of the CNN architectures, as well as the combination with recurrent neural networks (in order to include the temporal aspect of the physiological signals in the inference model), could potentially improve the performance of the current system, since such approaches have been successfully applied in other domains of application such as facial expression recognition [59-61]. Finally, the recorded video data provide an additional channel that can be integrated into the fusion architecture in order to improve the performance of the whole system. Therefore, the video modality should also be evaluated and assessed in combination with the physiological modalities.

In summary, the performed assessment suggests that deep learning approaches are relevant for the inference of pain intensity based on 1D physiological data, and such methods are able to significantly outperform traditional approaches based on hand-crafted features. Domain expert knowledge could be bypassed by enabling the designed deep architecture to learn relevant features from the data. In the future iterations of the current work, approaches consisting of combining both learned and hand-crafted features should be addressed. In addition, the designed architectures should be also assessed by replacing the classification experiments by regression experiments. Additionally, several data transformation approaches applied to the recorded 1D physiological data in order to generate 2D visual representations (e.g., spectrograms) should also be investigated in combination with established deep neural network approaches, specifically designed for this type of data representation.

Author Contributions: Conceptualisation, P.T. and F.S.; Methodology, P.T.; Software, P.T.; Validation, P.T. and P.B.; Formal Analysis, P.T.; Investigation, P.T. and F.S.; Writing-Original Draft Preparation, P.T. and P.B.; Writing-Review and Editing, P.T., P.B., H.A.K. and F.S.; Visualisation, P.T.; Supervision, H.A.K. and F.S.; Project Administration, H.A.K. and F.S.; and Funding Acquisition, H.A.K. and F.S.

Funding: The research leading to these results received funding from the Federal Ministry of Education and Research (BMBF, SenseEmotion) to F.S. (BMBF, e:Med, CONFIRM, ID 01ZX1708C) to H.A.K., and the Ministry of Science and Education Baden-Württemberg (Project ZIV) to H.A.K.

Acknowledgments: Peter Bellmann is supported by a scholarship of the Landesgraduiertenförderung Baden-Württemberg at Ulm University. We gratefully acknowledge the support of NVIDIA Corporation with the donation of the Tesla K40 GPU used for this research.

Conflicts of Interest: The authors declare no conflict of interest. 


\section{References}

1. LeCun, Y.; Bengio, Y.; Hinton, G. Deep Learning. Nature 2015, 521, 436-444. [CrossRef] [PubMed]

2. Bengio, Y.; Courville, A.; Vincent, P. Representation Learning: A Review and New Perspectives. IEEE Trans. Pattern Anal. Mach. Intell. 2013, 35, 1798-1828. [CrossRef] [PubMed]

3. Krizhevsky, A.; Sutskever, I.; Hinton, G.E. ImageNet Classification with Deep Convolutional Neural Networks. In Proceedings of the 25th International Conference on Neural Information Processing Systems, Lake Tahoe, NV, USA, 3-6 December 2012; pp. 1097-1105.

4. Simonyan, K.; Zisserman, A. Very Deep Convolution Networks for Large-Scale Image Recognition. In Proceedings of the 3rd IAPR Asian Conference on Pattern Recognition (ACPR), Kuala Lumpur, Malaysia, 3-6 November 2015; pp. 730-734.

5. Szegedy, C.; Liu, W.; Jia, Y.; Sermanet, P.; Reed, S.; Anguelov, D.; Erhan Dumitru abd Vanhoucke, V.; Rabinovich, A. Going deeper with convolutions. In Proceedings of the IEEE Conference on Computer Vision and Pattern Recognition (CVPR), Boston, MA, USA, 7-12 June 2015; pp. 1-9.

6. He, K.; Zhang, X.; Ren, S.; Sun, J.A. Deep Residual Learning for Image Recognition. In Proceedings of the IEEE Conference on Computer Vision and Pattern Recognition (CVPR), Las Vegas, NV, USA, 27-30 June 2016; pp. 770-778.

7. Mohd Kamarufin, J.A.; Abdullah, A.; Sallehuddin, R. A Review of Deep Learning Architectures and Their Application. In Modeling, Design and Simulation of Systems; Springer: Singapore, 2017; pp. 83-94.

8. Hinton, G.; Deng, L.; Yu, D.; Dahl, G.E.; Mohammed, A.r.; Jaitly, N.; Senior, A.; Nguyen, P.; Sainath, T.N.; Kingsbury, B. Deep Neural Networks for Acoustic Modeling in Speech Recognition: The Shared View of Four Research Groups. IEEE Signal Process. Mag. 2012, 29, 82-97. [CrossRef]

9. Zhang, Z.; Geiger, J.; Pohjalainen, J.; Mousa, A.E.D.; Jin, W.; Schuller, B. Deep Learning for Environmentallly Robust Speech Recognition: An Overview of Recent Developments. ACM Trans. Intell. Syst. Technol. 2018, 9, 49:1-49:28. [CrossRef]

10. Costa-jussà, M.R. From Feature to Paradigm: Deep Learning in Machine Translation. J. Artif. Intell. Res. 2018, 61, 947-974. [CrossRef]

11. Young, T.; Hazarika, D.; Poria, S.; Cambria, E. Recent Trends in Deep Learning Based Natural Language Processing [Review Article]. IEEE Comput. Intell. Mag. 2018, 13, 55-75. [CrossRef]

12. Martinez, H.P.; Bengio, Y.; Yannakakis, G.N. Learning Deep Physiological Models of Affect. IEEE Comput. Intell. Mag. 2013, 8, 20-33. [CrossRef]

13. LeCun, Y.; Kavukcuoglu, K.; Farabet, C. Convolutional networks and application in vision. In Proceedings of the IEEE International Symposium on Circuits and Systems, Paris, France, 30 May-2 June 2010; pp. 253-256.

14. Vincent, P.; Larochelle, H.; Bengio, Y.; Manzagol, P.A. Extracting and Composing Robust features with Denoising Autoencoders. In Proceedings of the 25th International Conference on Machine Learning, Helsinki, Finland, 5-9 July 2008; pp. 1096-1103.

15. Hecht-Nielsen, R. Theory of the backpropagation neural network. In Proceedings of the International Joint Conference on Neural Networks, Washington, DC, USA, 1989; pp. 593-605.

16. Zhong, Y.; Mengyuan, Z.; Yongxiong, W.; Jingdong, Y.; Jianhua, Z. Recognition of emotions using multimodal physiological signals and an ensemble deep learning model. Comput. Methods Programs Biomed. 2017, 140, 93-110.

17. Santamaria-Granados, L.; Munoz-Organero, M.; Ramirez-González, G.; Abdulhay, E.; Arunkumar, N. Using Deep Convolutional Neural Network for Emotion Detection on a Physiological Signals Dataset (AMIGOS). IEEE Access 2018, 7, 57-67. [CrossRef]

18. Kanjo, E.; Younis, E.M.; Ang, C.S. Deep learning analysis of mobile physiological, environmental and location sensor data for emotion detection. Inf. Fusion 2019, 49, 46-56. [CrossRef]

19. Hochreiter, S.; Schmidhuber, J. Long Short-Term Memory. Neural Comput. 1997, 9, 1735-1780. [CrossRef] [PubMed]

20. Geng, W.; Du, Y.; Jin, W.; Wei, W.; Hu, Y.; Li, J. Gesture recognition by instantaneous surface EMG images. Sci. Rep. 2016, 6, 36571. [CrossRef] [PubMed] 
21. Xing, K.; Ding, Z.; Jiang, S.; Ma, X.; Yang, K.; Yang, C.; Li, X.; Jiang, F. Hand Gesture Recognition Based on Deep Learning Method. In Proceedings of the IEEE Third International Conference on Data Science in Cyberspace, Guangzhou, China, 18-21 June 2018; pp. 542-546.

22. Zhai, X.; Jelfs, B.; Chan, R.H.M.; Tin, C. Self-Recalibrating Surface EMG Pattern Recognition for Neuroprosthesis Control Based on Convolutional Neural Network. Front. Neurosci. 2017, 11, 379. [CrossRef] [PubMed]

23. Zia ur Rehman, M.; Waris, A.; Gilani, S.O.; Jochumsen, M.; Niazi, I.K.; HJamil, M.; Farina, D.; Kamavuako, E.N. Multiday EMG-Based Classification of Hand Motions with Deep Learning Techniques. Sensors 2018, $18,2497$. [CrossRef]

24. Tayeb, Z.; Fedjaev, J.; Ghaboosi, N.; Richter, C.; Everding, L.; Qu, X.; Wu, Y.; Cheng, G.; Conradt, J. Validating Deep Neural Networks for Online Decoding of Motor Imagery Movements from EEG Signals. Sensors 2019, 19, 210. [CrossRef]

25. Faust, O.; Hagiwara, Y.; Hong, T.J.; Lih, O.S.; Acharya, U.R. Deep Learning for Healthcare Applications based on Physiological Signals: A Review. Comput. Methods Programs Biomed. 2018, 161, 1-13. [CrossRef]

26. Ganapathy, N.; Swaminathan, R.; Deserno, T.M. Deep Learning on 1-D Biosignals: A Taxonomy-based Survey. Yearb. Med. Inform. 2018, 27, 98-109. [CrossRef]

27. Lim, H.; Kim, B.; Noh, G.J.; Yoo, S.K. A Deep Neural Network-Based Pain Classifier Using a Photoplethysmography Signal. Sensors 2019, 19, 384. [CrossRef]

28. Abe, S. Support Vector Machines for Pattern Classification; Springer: London, UK, 2005.

29. Jiang, M.; Mieronkoski, R.; Syrjälä, E.; Anzanpour, A.; Terävä, V.; Rahmani, A.M.; Salanterä, S.; Aantaa, R.; Hagelberg, N.; Liljeberg, P. Acute pain intensity monitoring with the classification of multiple physiological parameters. J. Clin. Monit. Comput. 2019, 33, 493-507. [CrossRef]

30. Alazrai, R.; AL-Rawi, S.; Alwanni, H.; Daoud, M.I. Tonic Cold Pain Detection Using Choi-Williams Time-Frequency Distribution Analysis of EEG Signals: A Feasibility Study. Appl. Sci. 2019, 9, 3433. [CrossRef]

31. Thiam, P.; Kessler, V.; Amirian, M.; Bellmann, P.; Layher, G.; Zhang, Y.; Velana, M.; Gruss, S.; Walter, S.; Traue, H.C.; et al. Multi-modal Pain Intensity Recognition based on the SenseEmotion Database. IEEE Trans. Affect. Comput. 2019, 1. [CrossRef]

32. Thiam, P.; Schwenker, F. Multi-modal data fusion for pain intensity assessement and classification. In Proceedings of the 2017 Seventh International Conference on Image Processing Theory, Tools and Applications (IPTA), Montreal, QC, Canada, 28 November-1 December 2017; pp. 1-6.

33. Velana, M.; Gruss, S.; Layher, G.; Thiam, P.; Zhang, Y.; Schork, D.; Kessler, V.; Gruss, S.; Neumann, H.; Kim, J.; et al. The SenseEmotion Database: A Multimodal Database for the Development and Systematic Validation of an Automatic Pain- and Emotion-Recognition System. In Multimodal Pattern Recognition of Social Signals in Human-Computer-Interaction; Springer International Publishing: Cham, Switzerland, 2017; pp. 127-139.

34. Breiman, L. Random Forests. Mach. Learn. 2001, 45, 5-32. [CrossRef]

35. Kessler, V.; Thiam, P.; Amirian, M.; Schwenker, F. Pain recognition with camera photoplethysmography. In Proceedings of the Seventh International Conference on Image Processing Theory, Tools and Applications (IPTA), Montreal, QC, Canada, 28 November-1 December 2017; pp. 1-5.

36. Werner, P.; Al-Hamadi, A.; Niese, R.; Walter, S.; Gruss, S.; Traue, H.C. Automatic Pain Recognition from Video and Biomedical Signals. In Proceedings of the International Conference on Pattern Recognition (ICPR), Stockholm, Sweden, 24-28 August 2014; pp. 4582-4587.

37. Walter, S.; Gruss, S.; Limbrecht-Ecklundt, K.; Traue, H.C.; Werner, P.; Al-Hamadi, A.; Diniz, N.; Silva, G.M.; Andrade, A.O. Automatic Pain Quantification using Autonomic Parameters. Psychol. Neurosci. 2014, 7, 363-380. [CrossRef]

38. Gruss, S.; Treister, R.; Werner, P.; C. Traue, H.; Crawcour, S.; Andrade, A.; Walter, S. Pain Intensity Recognition Rates via Biopotential Feature Patterns with Support Vector Machines. PLoS ONE 2015, 10, 1-14. [CrossRef]

39. Walter, S.; Gruss, S.; Ehleiter, H.; Tan, J.; Traue, H.C.; Crawcour, S.; Werner, P.; Al-Hamadi, A.; Andrade, A. The BioVid heat pain database data for the advancement and systematic validation of an automated pain recognition system. In Proceedings of the IEEE International Conference on Cybernetics, Lausanne, Switzerland, 13-15 June 2013; pp. 128-131. 
40. Kächele, M.; Thiam, P.; Amirian, M.; Schwenker, F.; Palm, G. Methods for Person-Centered Continuous Pain Intensity Assessment From Bio-Physiological Channels. IEEE J. Sel. Top. Signal Process. 2016, 10, 854-864. [CrossRef]

41. Kächele, M.; Amirian, M.; Thiam, P.; Werner, P.; Walter, S.; Palm, G.; Schwenker, F. Adaptive Confidence Learning for the Personalization of Pain Intensity Estimation Systems. Evol. Syst. 2016, 8, 1-13. [CrossRef]

42. Bellmann, P.; Thiam, P.; Schwenker, F. Chapter Multi-classifier-Systems: Architectures, Algorithms and Applications. In Computational Intelligence for Pattern Recognition; Pedrycz, W., Chen, S.M., Eds.; Springer: Cham, Switzerland, 2018; Volume 777, pp. 83-113.

43. Breiman, L. Bagging Predictors. Mach. Learn. 1996, 24, 123-140. [CrossRef]

44. Freund, Y.; Schapire, R.E. A Decision-Theoretic Generalization of On-Line Learning and an Application to Boosting. J. Comput. Syst. Sci. 1997, 55, 119-139. [CrossRef]

45. LeCun, Y.; Boser, B.; Denker, J.S.; Henderson, D.; Howard, R.E.; Hubbard, W.; Jackel, L.D. Handwritten Digit Recognition with a Back-propagation Network. In Proceedings of the 2nd International Conference on Neural Information Processing Systems, Denver, CO, USA, 2-5 December 1990; pp. 396-404.

46. LeCun, Y.; Bottou, L.; Bengio, Y.; Haffner, P. Gradient-Based Learning Applied to Document Recognition. Proc. IEEE 1998, 86, 2278-2324. [CrossRef]

47. Ioffe, S.; Szegedy, C. Batch Normalization: Accelerating Deep Network Training by Reducing Internal Covariate Shift. In Proceedings of the 32nd International Conference on Machine Learning, Lille, France, 6-11 July 2015; pp. 448-456.

48. Srivastava, N.; Hinton, G.; Krizhevsky, A.; Sutskever, I.; Salakhutdinov, R. Dropout: A Simple Way to Prevent Neural Networks from Overfitting. J. Mach. Learn. Res. 2014, 15, 1929-1958.

49. Clevert, D.A.; Unterthiner, T.; Hochreiter, S. Fast and Accurate Deep Neural Network Learning by Exponential Linear Units (ELUs). In Proceedings of the 4th International Conference on Learning Representations (ICLR), San Juan, Puerto Rico, 2-4 May 2016.

50. Pyakillya, B.; Kazachenko, N.; Mikhailovsky, N. Deep Learning for ECG Classification. J. Phys. Conf. Ser. 2017, 913, 012004. [CrossRef]

51. Kingma, D.P.; Ba, J. Adam: A Method for Stochastic Optimization. In Proceedings of the 3rd International Conference on Learning Representations (ICLR), San Diego, CA, USA, 7-9 May 2015.

52. Keras: The Python Deep Learning Library. 2015. Available online: https:// keras.io (accessed on 16 October 2019).

53. Abadi, M.; Agarwal, A.; Barham, P.; Brevdo, E.; Chen, Z.; Citro, C.; Corrado, C.; Davis, A.; Dean, J.; Devin, M.; et al. TensorFlow: Large-Scale Machine Learning on Heterogeneous Systems. 2015. Available online: https://www. tensorflow.org/ (accessed on 16 October 2019).

54. Pedregosa, F.; Varoquaux, G.; Gramfort, A.; Michel, V.; Thirion, B.; Grisel, O.; Blondel, M.; Prettenhofer, P.; Weiss, R.; Dubourg, V.; et al. Scikit-learn: Machine Learning in Python. J. Mach. Learn. Res. 2011, 12, $2825-2830$.

55. Lopez-Martinez, D.; Picard, R. Continuous Pain Intensity Estimation from Autonomic Signals with Recurrent Neural Networks. In Proceedings of the 40th Annual International Conference of the IEEE Engineering in Medicine and Biology Society (EMBC), Honolulu, HI, USA, 18-21 July 2018; pp. 5624-5627.

56. Kächele, M.; Werner, P.; Walter, S.; Al-Hamadi, A.; Schwenker, F. Bio-Visual Fusion for Person-Independent Recognition of Pain Intensity. In Multiple Classifier Systems (MCS); Springer: Cham, Switzerland, 2015; pp. 220-230.

57. Kächele, M.; Thiam, P.; Amirian, M.; Werner, P.; Walter, S.; Schwenker, F.; Palm, G. Engineering Applications of Neural Networks. In Engineering Applications of Neural Networks, EANN 2015; Chapter Multimodal Data Fusion for Person-Independent, Continuous Estimation of Pain Intensity; Iliadis, L., Jayne, C., Eds.; Springer International Publishing: Cham, Switzerland, 2015; pp. 275-285.

58. Werner, P.; Al-Hamadi, A.; Limbrecht-Ecklundt, K.; Walter, S.; Gruss, S.; Traue, H.C. Automatic Pain Assessment with Facial Activity Descriptors. IEEE Trans. Affect. Comput. 2017, 8, 286-299. [CrossRef] 
59. Jung, H.; Lee, S.; Yim, J.; Park, S.; Kim, J. Joint Fine-Tuning in Deep Neural Networks for Facial Expression. In Proceedings of the IEEE International Conference on Computer Vision (ICCV), Santiago, Chile, 7-13 December 2015; pp. 2983-2991.

60. Rodriguez, P.; Cucurull, G.; Gonzàlez, J.; Gonfaus, J.M.; Nasrollahi, K.; Moeslund, T.B.; Roca, F.X. Deep Pain: Exploiting Long Short-Term Memory Networks for Facial Expression Classification. IEEE Trans. Cybern. 2018, 1-11. [CrossRef]

61. Yan, J.; Zheng, W.; Vui, Z.; Song, P. A Joint Convolutional Bidirectional LSTM Framework for Facial Expression Recognition. IEICE Trans. Inf. Syst. 2018, E101.D, 1217-1220. [CrossRef]

(C) 2019 by the authors. Licensee MDPI, Basel, Switzerland. This article is an open access article distributed under the terms and conditions of the Creative Commons Attribution (CC BY) license (http:/ / creativecommons.org/licenses/by/4.0/). 